Proyecciones Journal of Mathematics

Vol. 38, No 1, pp. 177-189, March 2019.

Universidad Católica del Norte

Antofagasta - Chile

\title{
Even vertex equitable even labeling for snake related graphs
}

\author{
A. Lourdusamy \\ St. Xavier's College, India \\ S. Jenifer Wency \\ Manonmaniam Sundaranar University, India \\ and \\ F. Patrick \\ St. Xavier's College, India \\ Received : April 2018. Accepted : August 2018
}

\begin{abstract}
Let $G$ be a graph with $p$ vertices and $q$ edges and $A=$ $\{0,2,4, \cdots, q+1\}$ if $q$ is odd or $A=\{0,2,4, \cdots, q\}$ if $q$ is even. A graph $G$ is said to be an even vertex equitable even labeling if there exists a vertex labeling $f: V(G) \rightarrow A$ that induces an edge labeling $f^{*}$ defined by $f^{*}(u v)=f(u)+f(v)$ for all edges uv such that for all $a$ and $b$ in $A,\left|v_{f}(a)-v_{f}(b)\right| \leq 1$ and the induced edge labels are $2,4, \cdots, 2 q$, where $v_{f}(a)$ be the number of vertices $v$ with $f(v)=a$ for $a \in A$. A graph that admits even vertex equitable even labeling is called an even vertex equitable even graph. In this paper, we prove that $S\left(D\left(Q_{n}\right)\right)$, $S\left(D\left(T_{n}\right)\right), D A\left(Q_{m}\right) \odot n K_{1}, D A\left(T_{m}\right) \odot n K_{1}, S\left(D A\left(Q_{n}\right)\right)$ and $S\left(D A\left(T_{n}\right)\right)$ are an even vertex equitable even graphs.
\end{abstract}

AMS Subject Classification (2010): 05C78.

Keywords: vertex equitable labeling, even vertex equitable even labeling, double quadrilateral snake, double triangular snake. 


\section{Introduction}

All graphs considered here are simple, finite, connected and undirected. The vertex set and the edge set of a graph are denoted by $V(G)$ and $E(G)$ respectively. We follow the basic notations and terminology of graph theory as in [2]. A labeling of a graph is a map that carries the graph elements to the set of numbers, usually to the set of non-negative or positive integers. If the domain is the set of vertices then the labeling is called vertex labeling. If the domain is the set of edges then the labeling is called edge labeling. If the labels are assigned to both vertices and edges then the labeling is called total labeling. For a dynamic survey of various graph labeling, we refer to Gallian [1].

Lourdusamy et al. introduced the concept of vertex equitable labeling in [15]. Let $G$ be a graph with $p$ vertices and $q$ edges and let $A=\left\{0,1,2, \cdots,\left\lceil\frac{q}{2}\right\rceil\right\}$. A vertex labeling $f: V(G) \rightarrow A$ induces an edge labeling $f^{*}$ defined by $f^{*}(u v)=f(u)+f(v)$ for all edges $u v$. For $a \in A$, let $v_{f}(a)$ be the number of vertices $v$ with $f(v)=a$. A graph $G$ is said to be vertex equitable if there exists a vertex labeling $f$ such that for all $a$ and $b$ in $A,\left|v_{f}(a)-v_{f}(b)\right| \leq 1$ and the induced edge labels are $1,2,3, \cdots, q$.

Motivated by the concept of vertex equitable labeling and further results by Jeyanthi et al. in [3, 4, 5, 6, 7, 8, 9, 10, 11]. Lourdusamy et al. introduced the concept of even vertex equitable even labeling [16] and further studied in $[12,13,14,16,17]$.

In this paper, we prove that $S\left(D\left(Q_{n}\right)\right), S\left(D\left(T_{n}\right)\right), D A\left(Q_{m}\right) \odot$ $n K_{1}, D A\left(T_{m}\right) \odot n K_{1}, S\left(D A\left(Q_{n}\right)\right)$ and $S\left(D A\left(T_{n}\right)\right)$ admit an even vertex equitable even labeling. We use the following definitions in the subsequent sections.

Definition 1.1. Let $G$ be a graph with $p$ vertices and $q$ edges and $A=\{0,2,4, \cdots, q+1\}$ if $q$ is odd or $A=\{0,2,4, \cdots, q\}$ if $q$ is even. $A$ graph $G$ is said to be an even vertex equitable even labeling if there exists a vertex labeling $f: V(G) \rightarrow A$ that induces an edge labeling $f^{*}$ defined by $f^{*}(u v)=f(u)+f(v)$ for all edges $u v$ such 
that for all $a$ and $b$ in $A,\left|v_{f}(a)-v_{f}(b)\right| \leq 1$ and the induced edge labels are $2,4, \cdots, 2 q$, where $v_{f}(a)$ be the number of vertices $v$ with $f(v)=a$ for $a \in A$. A graph that admits even vertex equitable even labeling is called an even vertex equitable even graph.

Definition 1.2. The double quadrilateral snake $D\left(Q_{n}\right)$ is a graph obtained from a path $P_{n}$ with vertices $u_{1}, u_{2}, \cdots, u_{n}$ by joining $u_{i}$ and $u_{i+1}$ to the new vertices $v_{i}, x_{i}$ and $w_{i}, y_{i}$ respectively and then joining $v_{i}, w_{i}$ and $x_{i}, y_{i}$ for $i=1,2, \cdots, n-1$.

Definition 1.3. The double triangular snake $D\left(T_{n}\right)$ is a graph obtained from a path $P_{n}$ with vertices $v_{1}, v_{2}, \cdots, v_{n}$ by joining $v_{i}$ and $v_{i+1}$ to the new vertices $w_{i}$ and $u_{i}$ for $i=1,2, \cdots, n-1$.

Definition 1.4. A double alternate quadrilateral snake $D A\left(Q_{n}\right)$ consists of two alternate quadrilateral snakes that have a common path. That is, a double alternate quadrilateral snake is obtained from a path $u_{1}, u_{2}, \cdots, u_{n}$ by joining $u_{i}$ and $u_{i+1}$ (alternately) to the two new vertices $v_{i}, x_{i}$ and $w_{i}, y_{i}$ respectively and adding the edges $v_{i} w_{i}$ and $x_{i} y_{i}$ for $i=1,2, \cdots, n-1$.

Definition 1.5. A double alternate triangular snake $D A\left(T_{n}\right)$ consists of two alternate triangular snakes that have a common path. That is, a double alternate triangular snake is obtained from a path $u_{1}, u_{2}, \cdots, u_{n}$ by joining $u_{i}$ and $u_{i+1}$ (alternately) to the two new vertices $v_{i}$ and $w_{i}$ for $i=1,2, \cdots, n-1$.

Definition 1.6. Let $G$ be a graph. The subdivision graph $S(G)$ is obtained from $G$ by subdividing each edge of $G$ with a vertex.

Definition 1.7. The corona $G_{1} \odot G_{2}$ of two graphs $G_{1}\left(p_{1}, q_{1}\right)$ and $G_{2}\left(p_{2}, q_{2}\right)$ is defined as the graph obtained by taking one copy of $G_{1}$ and $p_{1}$ copies of $G_{2}$ and joining the $i^{\text {th }}$ vertex of $G_{1}$ to every vertex in the $i^{\text {th }}$ copy of $G_{2}$.

\section{Main Results}

Theorem 2.1. Let $G_{1}\left(p_{1}, q_{1}\right), G_{2}\left(p_{2}, q_{2}\right), \cdots, G_{m}\left(p_{m}, q_{m}\right)$ be an even vertex equitable even graphs with $\sum_{i=1}^{m} q_{i}$ is even and let $u_{i}, v_{i}$ be 
the vertices of $G_{i}(1 \leq i \leq m)$ labeled by 0 and $q_{i}$. Then the graph $G$ obtained by identifying $v_{1}$ with $u_{2}$ and $v_{2}$ with $u_{3}$ and $v_{3}$ with $u_{4}$ and so on until we identify $v_{m-1}$ with $u_{m}$ is also an even vertex equitable even labeling.

Proof. Let $f_{i}$ be an even vertex equitable even labeling of a graph $G_{i}$ of order $p_{1}+p_{2}+\cdots+p_{m}-(m-1)$ and size $\sum_{i=1}^{m} q_{i}$. We define a vertex labeling $h$ of $G$ such that $h(x)=f_{1}(x), x \in V\left(G_{1}\right)$, $h(x)=f_{i}(x)+\sum_{i=1}^{m} q_{i}, x \in V\left(G_{i}\right), 2 \leq i \leq m$.

Then the induced edge labels of $G_{i}$ are $2,4, \cdots, 2 q_{1}, 2 q_{1}+2,2 q_{1}+$ $4, \cdots, 2 q_{1}+2 q_{2}, 2 q_{1}+2 q_{2}+2,2 q_{1}+2 q_{2}+4, \cdots, 2 q_{1}+2 q_{2}+2 q_{3}, \cdots$, $2 \sum_{i=1}^{m-1} q_{i}+2,2 \sum_{i=1}^{m-1} q_{i}+4, \cdots, 2 \sum_{i=1}^{m} q_{i}$. It can be verified that the induced edge labels of $G$ are distinct and $\left|v_{f}(a)-v_{f}(b)\right| \leq 1$ for all $a, b \in A$. Thus, $G$ admits an even vertex equitable even labeling.

Theorem 2.2. The graph $S\left(D\left(Q_{n}\right)\right)$ admits an even vertex equitable even labeling.

Proof. An even vertex equitable even labeling of $S\left(D\left(Q_{2}\right)\right)$ is shown in Figure 1. Let $G_{i}=S\left(D\left(Q_{2}\right)\right)$ for $1 \leq i \leq n-1$. By Theorem 2.1, $S\left(D\left(Q_{n}\right)\right)$ admits an even vertex equitable even labeling.

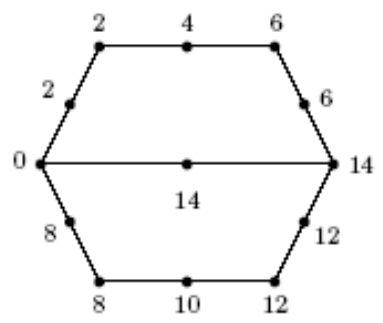

Figure 1

Theorem 2.3. The graph $S\left(D\left(T_{n}\right)\right)$ admits an even vertex equitable even labeling. 
Proof. An even vertex equitable even labeling of $S\left(D\left(T_{2}\right)\right)$ is shown in Figure 2. Let $G_{i}=S\left(D\left(T_{2}\right)\right)$ for $1 \leq i \leq n-1$. By Theorem 2.1, $S\left(D\left(T_{n}\right)\right)$ admits an even vertex equitable even labeling.

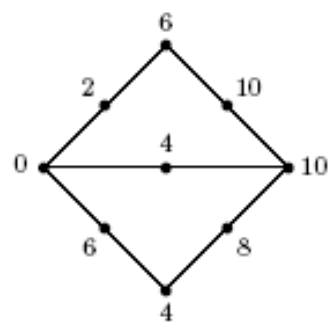

Figure 2

Theorem 2.4. Let $G_{1}\left(p_{1}, q\right), G_{2}\left(p_{2}, q\right), \cdots, G_{m}\left(p_{m}, q\right)$ be an even vertex equitable even graphs with $q$ odd and $u_{i}, v_{i}$ be vertices of $G_{i}(1 \leq i \leq m)$ labeled by 0 and $q$. Then the graph $G$ obtained by joining $v_{1}$ with $u_{2}$ and $v_{2}$ with $u_{3}$ and $v_{3}$ with $u_{4}$ and so on until joining $v_{m-1}$ with $u_{m}$ by an edge is also an even vertex equitable even labeling.

Proof. The graph $G$ has $p_{1}+p_{2}+\cdots+p_{m}$ vertices and $m q+(m-1)$ edges. Let $f_{i}$ be an even vertex equitable even labeling of $G_{i}(1 \leq i \leq$ $m)$. Define a vertex labeling $f: V(G) \rightarrow A=\{0,2, \cdots, m q+(m-1)\}$ by $f(x)=f_{i}(x)+(i-1)(q+1)$ if $x \in G_{i}$ for $1 \leq i \leq m$. The edge labels of $G_{i}$ are increased by $2(i-1)(q+1)$ for $i=1,2, \cdots, m$ under the new labeling $f$. The bridge between the two graphs $G_{i}, G_{i+1}$ will get the label $2 i(q+1), 1 \leq i \leq m-1$. It can be verified that the induced edge labels of $G$ are $2,4, \cdots, 2(m q+m-1)$ and $\left|v_{f}(a)-v_{f}(b)\right| \leq 1$ for all $a, b \in A$. Thus, $G$ admits an even vertex equitable even labeling.

Theorem 2.5. The graph $D A\left(Q_{2}\right) \odot n K_{1}$ admits an even vertex equitable even labeling.

Proof. Let $V\left(D A\left(Q_{2}\right) \odot n K_{1}\right)=\left\{u_{1}, u_{2}, v, w, x, y\right\} \bigcup\left\{u_{i, j}: 1 \leq\right.$ $i \leq 2,1 \leq j \leq n\} \bigcup\left\{v_{j}, w_{j}, x_{j}, y_{j}: 1 \leq j \leq n\right\}$ and 
$E\left(D A\left(Q_{2}\right) \odot n K_{1}\right)=\left\{u_{i} u_{i, j}: 1 \leq i \leq 2,1 \leq j \leq n\right\}$

$\bigcup\left\{u_{1} u_{2}, u_{1} v, v w, w u_{2}, u_{1} x, x y, y u_{2}\right\} \bigcup\left\{v v_{j}, w w_{j}, x x_{j}, y y_{j}: 1 \leq j \leq\right.$ $n\}$. Then $D A\left(Q_{2}\right) \odot n K_{1}$ has $6(n+1)$ vertices and $6 n+7$ edges.

Define a vertex labeling $f: V\left(D A\left(Q_{2}\right) \odot n K_{1}\right) \rightarrow A=\{0,2,4, \cdots, 6 n+$ 8\} as follows: $f\left(u_{1}\right)=0, f\left(u_{2}\right)=6 n+8, f(v)=2 n+2, f(w)=$ $4(n+1), f(x)=2 n+4, f(y)=4(n+2), f\left(u_{1, j}\right)=2 j$ if $1 \leq j \leq n$, $f\left(v_{j}\right)=2 j+2$ if $1 \leq j \leq n, f\left(y_{j}\right)=2 n+4+2 j$ if $1 \leq j \leq n$, $f\left(u_{2, j}\right)=6 n+8-2 j$ if $1 \leq j \leq n-1, f\left(u_{2, n}\right)=f\left(x_{n}\right)=4 n+6$, $f\left(w_{1}\right)=2, f\left(w_{j}\right)=6 n+10-2 j$ if $2 \leq j \leq n$ and $f\left(x_{j}\right)=2 n+2 j+2$ if $1 \leq j \leq n-1$. It can be verified that the induced edge labels of $D A\left(Q_{2}\right) \odot n K_{1}$ are $2,4, \cdots, 12 n+14$ and $\left|v_{f}(a)-v_{f}(b)\right| \leq 1$ for all $a, b \in A$. Thus, $D A\left(Q_{2}\right) \odot n K_{1}$ admits an even vertex equitable even labeling.

An even vertex equitable even labeling of $D A\left(Q_{2}\right) \odot 3 K_{1}$ is shown in Figure 3.

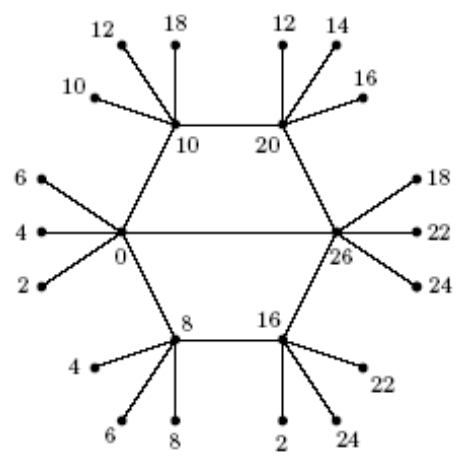

FIGURE 3

Theorem 2.6. The graph $D A\left(Q_{m}\right) \odot n K_{1}$ admits an even vertex equitable even labeling.

Proof. By Theorem 2.5, DA(Q $\left.Q_{2}\right) \odot n K_{1}$ is an even vertex equitable even graph. Let $G_{i}=D A\left(Q_{2}\right) \odot n K_{1}$ for $1 \leq i \leq m-1$. Since each $G_{i}$ has $6 n+7$ edges, by Theorem 2.4,DA( $\left.Q_{m}\right) \odot n K_{1}$ admits an even vertex equitable even labeling. 
Theorem 2.7. The graph $D A\left(T_{2}\right) \odot n K_{1}$ admits an even vertex equitable even labeling.

Proof. Let $V\left(D A\left(T_{2}\right) \odot n K_{1}\right)=\left\{u_{1}, u_{2}, u, w\right\} \bigcup\left\{u_{i, j}: 1 \leq i \leq\right.$ $2,1 \leq j \leq n\} \bigcup\left\{v_{j}, w_{j}: 1 \leq j \leq n\right\}$ and $E\left(D A\left(T_{2}\right) \odot n K_{1}\right)=\left\{u_{i} u_{i, j}:\right.$ $1 \leq i \leq 2,1 \leq j \leq n\} \bigcup\left\{u_{1} u_{2}, u_{1} v, v u_{2}, u_{1} w, u_{1} w, w u_{2}\right\} \bigcup\left\{v v_{j}, w w_{j}:\right.$ $1 \leq j \leq n\}$. Then $D A\left(T_{2}\right) \odot n K_{1}$ has $4(n+1)$ vertices and $4 n+5$ edges.

Define a vertex labeling $f: V\left(D A\left(T_{2}\right) \odot n K_{1}\right) \rightarrow A=\{0,2,4, \cdots, 8 n+$ 10\} as follows: $f\left(u_{1}\right)=0, f\left(u_{2}\right)=4 n+6, f(v)=2 n+2, f(w)=$ $2 n+4, f\left(u_{1, j}\right)=2 j, f\left(v_{j}\right)=2 j+2, f\left(y_{j}\right)=2 n+4+2 j, f\left(u_{2, j}\right)=$ $4 n+6-2 j$, for $1 \leq j \leq n$. It can be verified that the induced edge labels of $D A\left(T_{2}\right) \odot n K_{1}$ are $2,4, \cdots, 8 n+10$ and $\left|v_{f}(a)-v_{f}(b)\right| \leq 1$ for all $a, b \in A$. Thus, $D A\left(T_{2}\right) \odot n K_{1}$ admits an even vertex equitable even labeling.

An even vertex equitable even labeling of $D A\left(T_{2}\right) \odot 3 K_{1}$ is shown in Figure 4.

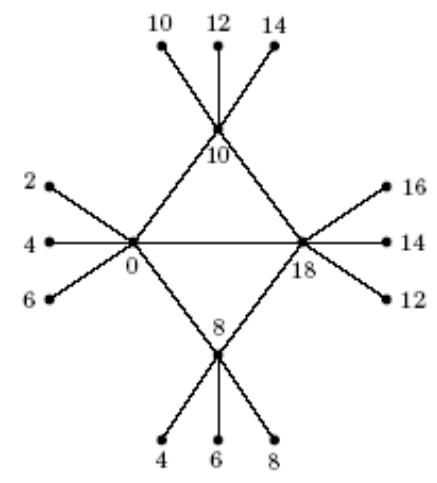

Figure 4

Theorem 2.8. The graph $D A\left(T_{m}\right) \odot n K_{1}$ admits an even vertex equitable even labeling.

Proof. By Theorem 2.7, $D A\left(T_{2}\right) \odot n K_{1}$ is an even vertex equitable even graph. Let $G_{i}=D A\left(T_{2}\right) \odot n K_{1}$ for $1 \leq i \leq m-1$. Since each $G_{i}$ has $4 n+5$ edges, by Theorem $2.4, D A\left(T_{m}\right) \odot n K_{1}$ admits an even vertex equitable even labeling. 
Theorem 2.9. The graph $S\left(D A\left(Q_{n}\right)\right)$ is an even vertex equitable even graph.

Proof. Let $G=S\left(D A\left(Q_{n}\right)\right)$. Let $u_{1}, u_{2}, \cdots, u_{n}$ be the vertices of the path $P_{n}$. We construct $D A\left(Q_{n}\right)$ by joining every $u_{2 i-1}$ is adjacent to $v_{i}, x_{i}$ and $u_{2 i}$ is adjacent to $w_{i}, y_{i}$ and also $v_{i}, x_{i}$ is adjacent to $w_{i}, y_{i}$ respectively for $1 \leq i \leq\left\lfloor\frac{n}{2}\right\rfloor$. Let $V(G)=V\left(D A\left(Q_{n}\right)\right) \bigcup\left\{u_{i}^{\prime}\right.$ : $1 \leq i \leq n-1\} \bigcup\left\{v_{i}^{\prime}, w_{i}^{\prime}, x_{i}^{\prime}, y_{i}^{\prime}, z_{i}, z_{i}^{\prime}: 1 \leq i \leq\left\lfloor\frac{n}{2}\right\rfloor\right\}$ and $E(G)=$ $\left\{u_{i} u_{i}^{\prime}, u_{i}^{\prime} u_{i+1}: 1 \leq i \leq n-1\right\} \bigcup\left\{v_{i} v_{i}^{\prime}, v_{i} z_{i}, z_{i} w_{i}, w_{i} w_{i}^{\prime}, x_{i} x_{i}^{\prime}, x_{i} z_{i}^{\prime}, z_{i}^{\prime} y_{i}, y_{i} y_{i}^{\prime}:\right.$ $\left.1 \leq i \leq\left\lfloor\frac{n}{2}\right\rfloor\right\} \bigcup\left\{u_{2 i-1} v_{i}^{\prime}, u_{2 i-1} x_{i}^{\prime}, u_{2 i} w_{i}^{\prime}, u_{2 i} y_{i}^{\prime}: 1 \leq i \leq\left\lfloor\frac{n}{2}\right\rfloor\right\}$. Then

$$
|V(G)|=\{7 n-6 \text { if } n \text { is odd } 7 n-1 \text { if } n \text { is even , }
$$

$$
|E(G)|=\{8 n-8 \text { if } n \text { is odd } 8 n-2 \text { if } n \text { is even. }
$$

Case (i): $n$ is even.

Define $f: V(G) \rightarrow A=\{0,2, \cdots, 8 n-2\}$ as follows:

$$
\begin{aligned}
& f\left(u_{2 i-1}\right)=16(i-1), 1 \leq i \leq \frac{n}{2} ; \\
& f\left(u_{2 i}\right)=f\left(u_{2 i-1}^{\prime}\right)=16 i-2,1 \leq i \leq \frac{n}{2} ; \\
& f\left(u_{2 i}^{\prime}\right)=16 i, 1 \leq i \leq \frac{n-2}{2} ; \\
& f\left(v_{i}\right)=f\left(v_{i}^{\prime}\right)=16 i-8,1 \leq i \leq \frac{n}{2} \\
& f\left(w_{i}\right)=f\left(w_{i}^{\prime}\right)=16 i-4,1 \leq i \leq \frac{n}{2} \\
& f\left(x_{i}\right)=f\left(x_{i}^{\prime}\right)=16 i-14,1 \leq i \leq \frac{n}{2} \\
& f\left(y_{i}\right)=f\left(y_{i}^{\prime}\right)=16 i-10,1 \leq i \leq \frac{n}{2} \\
& f\left(z_{i}\right)=16 i-6,1 \leq i \leq \frac{n}{2} \\
& f\left(z_{i}^{\prime}\right)=16 i-12,1 \leq i \leq \frac{n}{2}
\end{aligned}
$$

It can be verified that the induced edge labels of $S\left(D A\left(Q_{n}\right)\right)$ are $2,4, \cdots, 16 n-4$ and $\left|v_{f}(a)-v_{f}(b)\right| \leq 1$ for all $a, b \in A$. Hence, $S\left(D A\left(Q_{n}\right)\right)$ is an even vertex equitable even graph.

Case (ii): $n$ is odd.

We define a vertex labeling $f: V(G) \rightarrow A=\{0,2, \cdots, 8 n-8\}$ as follows. Assign the labels to the vertices $u_{2 i-1}\left(1 \leq i \leq\left\lceil\frac{n}{2}\right\rceil\right)$ and 
$u_{2 i}, u_{2 i-1}^{\prime}, u_{2 i}^{\prime}, v_{i}, v_{i}^{\prime}$

$w_{i}, w_{i}^{\prime}, x_{i}, x_{i}^{\prime}, y_{i}, y_{i}^{\prime}, z_{i}, z_{i}^{\prime}\left(1 \leq i \leq\left\lfloor\frac{n}{2}\right\rfloor\right)$ as in Case (i). It can be verified that the induced edge labels of $S\left(D A\left(Q_{n}\right)\right)$ are $2,4, \cdots, 16 n-16$ and $\left|v_{f}(a)-v_{f}(b)\right| \leq 1$ for all $a, b \in A$. Hence, $S\left(D A\left(Q_{n}\right)\right)$ is an even vertex equitable even graph.

An even vertex equitable even labeling of $S\left(D A\left(Q_{6}\right)\right)$ is shown in Figure 5.

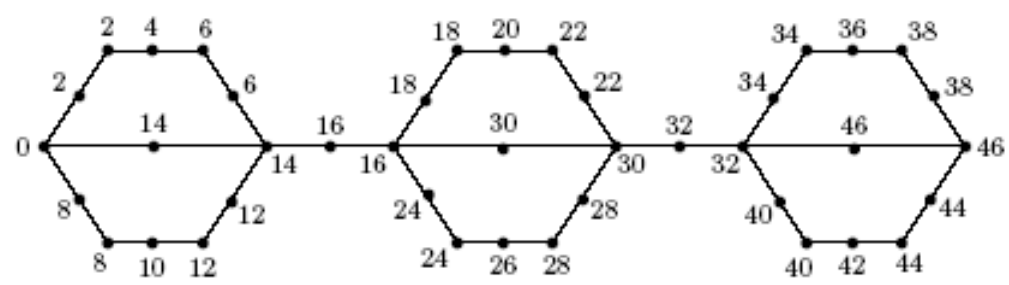

Figure 5

Theorem 2.10. The graph $S\left(D A\left(T_{n}\right)\right)$ is an even vertex equitable even graph.

Proof. Let $G=S\left(D A\left(T_{n}\right)\right)$. Let $u_{1}, u_{2}, \cdots, u_{n}$ be the vertices of the path $P_{n}$. We construct $D A\left(T_{n}\right)$ by joining every $u_{2 i-1}, u_{2 i}$ to the new vertices $v_{i}, w_{i}$ for $1 \leq i \leq\left\lfloor\frac{n}{2}\right\rfloor$. Let $V(G)=V\left(D A\left(T_{n}\right)\right) \bigcup\left\{u_{i}^{\prime}\right.$ : $1 \leq i \leq n-1\} \bigcup\left\{x_{i}, y_{i}, x_{i}^{\prime}, y_{i}^{\prime}: 1 \leq i \leq\left\lfloor\frac{n}{2}\right\rfloor\right\}$ and $E(G)=\left\{u_{i} u_{i}^{\prime}, u_{i}^{\prime} u_{i+1}\right.$ : $1 \leq i \leq n-1\} \bigcup\left\{x_{i} v_{i}, x_{i}^{\prime} w_{i}, v_{i} y_{i}, w_{i} y_{i}^{\prime}: 1 \leq i \leq\left\lfloor\frac{n}{2}\right\rfloor\right\}$ $\bigcup\left\{u_{2 i-1} x_{i}, u_{2 i-1} x_{i}^{\prime}, u_{2 i} y_{i}, u_{2 i} y_{i}^{\prime}: 1 \leq i \leq\left\lfloor\frac{n}{2}\right\rfloor\right\}$. Then

$$
\begin{aligned}
& |V(G)|= \begin{cases}5 n-4 & \text { if } n \text { is odd } \\
5 n-1 & \text { if } n \text { is even, }\end{cases} \\
& |E(G)|= \begin{cases}6 n-6 & \text { if } n \text { is odd } \\
6 n-2 & \text { if } n \text { is even. }\end{cases}
\end{aligned}
$$


Case (i): $n$ is even.

Define $f: V(G) \rightarrow A=\{0,2, \cdots, 6 n-2\}$ as follows:

$$
\begin{aligned}
& f\left(u_{2 i-1}\right)=12(i-1), 1 \leq i \leq \frac{n}{2} ; \\
& f\left(u_{2 i}\right)=f\left(y_{i}\right)=12 i-2,1 \leq i \leq \frac{n}{2} ; \\
& f\left(u_{2 i-1}^{\prime}\right)=12 i-10,1 \leq i \leq \frac{n}{2} ; \\
& f\left(u_{2 i}^{\prime}\right)=12 i, 1 \leq i \leq \frac{n-2}{2} ; \\
& f\left(v_{i}\right)=12 i-4,1 \leq i \leq \frac{n}{2} ; \\
& f\left(x_{i}\right)=f\left(y_{i}^{\prime}\right)=12 i-6,1 \leq i \leq \frac{n}{2} ; \\
& f\left(w_{i}\right)=f\left(x_{i}^{\prime}\right)=12 i-8,1 \leq i \leq \frac{n}{2} .
\end{aligned}
$$

It can be verified that the induced edge labels of $S\left(D A\left(T_{n}\right)\right)$ are $2,4, \cdots, 12 n-4$ and $\left|v_{f}(a)-v_{f}(b)\right| \leq 1$ for all $a, b \in A$. Hence, $S\left(D A\left(T_{n}\right)\right)$ is an even vertex equitable even graph.

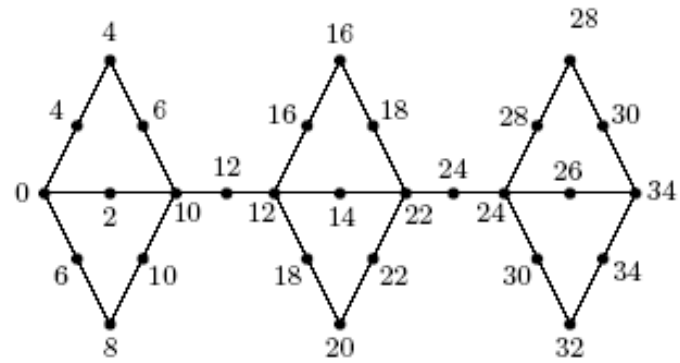

Figure 6

Case (ii): $n$ is odd.

We define a vertex labeling $f: V(G) \rightarrow A=\{0,2, \cdots, 6 n-6\}$ as follows. Assign the labels to the vertices $u_{2 i-1}\left(1 \leq i \leq\left\lceil\frac{n}{2}\right\rceil\right)$ and $u_{2 i}, u_{2 i-1}^{\prime}, u_{2 i}^{\prime}, v_{i}, w_{i}$,

$x_{i}, x_{i}^{\prime}, y_{i}, y_{i}^{\prime}\left(1 \leq i \leq\left\lfloor\frac{n}{2}\right\rfloor\right)$ as in Case (i). It can be verified that the induced edge labels of $S\left(D A\left(T_{n}\right)\right)$ are $2,4, \cdots, 12 n-12$ and $\mid v_{f}(a)-$ $v_{f}(b) \mid \leq 1$ for all $a, b \in A$. Hence, $S\left(D A\left(T_{n}\right)\right)$ is an even vertex equitable even graph.

An even vertex equitable even labeling of $S\left(D A\left(T_{6}\right)\right)$ is shown in Figure 6. 


\section{References}

[1] J. A. Gallian, A Dynamic Survey of Graph Labeling, The Electronic Journal of Combinatorics, 19, (2016).

[2] F. Harary, Graph Theory, Addison-wesley, Reading, Mass, (1972).

[3] P. Jeyanthi and A. Maheswari, Some Results on Vertex Equitable Labeling, Open Journal of Discrete Mathematics, 2(2), pp. 51-57, (2012).

[4] P. Jeyanthi and A. Maheswari, Vertex Equitable Labeling of Transformed Trees, Journal of Algorithms and Computation, 44 (1), pp. 9-20, (2013).

[5] P. Jeyanthi, A. Maheswari and M. Vijaya Laksmi, Vertex Equitable Labeling of Cycle and Star Related graphs, Journal of Scientific Research, 7(3), pp. 33-42, (2015).

[6] P. Jeyanthi and A. Maheswari, Vertex Equitable Labeling of Cycle and Path related graphs, Utilitas Mathematica, 98, pp. 215-226, (2015).

[7] P. Jeyanthi A. Maheswari and M. Vijaya Laksmi, Vertex Equitable Labeling of Double Alternate Snake Graphs,Journal of Algorithms and Computation, 46, pp. 27-34, (2015).

[8] P. Jeyanthi, A. Maheswari and M. Vijaya Laksmi, Vertex Equitable Labeling of Super Subdivision Graphs, Scientific International, 27 (5), pp. 3881-3883, (2015).

[9] P. Jeyanthi, A. Maheswari and M. Vijaya Laksmi, New Results on Vertex Equitable labeling, Journal of Algebra Combinatorics Discrete Structures and Applications, 3 (2), pp. 97-104, (2016).

[10] P. Jeyanthi, A. Maheswari and M. Vijaya Laksmi, Vertex Equitable Labeling of Union of Cyclic Snake graphs, Proyecciones Journal of Mathematics, 35 (1), pp. 177-186, (2016). 
[11] P. Jeyanthi, A. Maheswari and M. Vijaya Laksmi, Odd Vertex Equitable Even Labeling of Graphs, Proyecciones Journal of Mathematics, 36 (1), pp. 1-11, (2017).

[12] A. Lourdusamy and F. Patrick, Even Vertex Equitable Even Labeling of Path and Bistar Related Graphs, Information Sciences and Computing, Article ID: ISC650716, pp. 1-9, (2016).

[13] A. Lourdusamy and F. Patrick, Even Vertex Equitable Even Labeling for Cycle Related Graphs, Kragujevac Journal of Mathematics, 43 (3), pp. 427-441, (2019).

[14] A. Lourdusamy and F. Patrick, On Even Vertex Equitable Even Graphs, International Journal of Pure and Applied Mathematics, 117 (12), pp. 19-25, (2017).

[15] A. Lourdusamy and M. Seenivasan, Vertex Equitable Labeling of Graphs, Journal of Discrete Mathematical Sciences and Cryptography, 11 (6), pp. 727-735, (2008).

[16] A. Lourdusamy, J. Shobana Mary and F. Patrick, Even Vertex Equitable Even Labeling, Sciencia Acta Xaveriana, 7 (1), pp. 37-46, (2016).

[17] A. Lourdusamy, J. Shobana Mary and F. Patrick, Even Vertex Equitable Even Labeling for Path Related Graphs, Sciencia Acta Xaveriana, 7 (1), pp. 47-56, (2016).

\author{
A. Lourdusamy \\ Department of Mathematics, \\ St. Xavier's College, \\ Palayamkottai, Tamil Nadu, \\ India \\ e-mail : lourdusamy15@gmail.com
}


Even vertex equitable even labeling for snake related graphs 189

\section{S. Jenifer Wency}

Reg. No. 17211282092013,

Department of Mathematics,

St. Xavier's College, Palayamkottai, Manonmaniam Sundaranar University,

Abishekapatti, Tirunelveli - 627 012,

Tamil Nadu,

India

e-mail : jeniferwency@gmail.com

and

\section{F. Patrick}

Department of Mathematics,

St. Xavier's College,

Palayamkottai, Tamil Nadu,

India

e-mail : patrick881990@gmail.com 